\title{
Ethical Oversight of Implementation Research In Rural Settings Of A Developing Country During The COVID- 19 Outbreak
}

\author{
Kyaw Lwin Show ${ }^{1}$, Theingi Thwin ${ }^{2}$, Nyein Su Aye ${ }^{3}$, Zaw Than Htun ${ }^{4}$, Khin Thet Wai ${ }^{5}$ \\ ${ }^{1}$ Research Officer, Health Systems Research Division Member, Institutional Review Board \\ (IRB), Department of Medical Research. (DMR) \\ ${ }^{2}$ Director (Retd.), Member, IRB, DMR \\ ${ }^{3}$ Research Officer, Health Systems Research Division Member, IRB, DMR \\ ${ }^{4}$ Director General cum Vice-Chair Institutional Review Board (IRB), Department of Medical \\ Research. (DMR) \\ ${ }^{5}$ Institutional Review Board (IRB), Department of Medical Research. (DMR) \\ No. 5, Ziwaka Road, Dagon Township, Yangon 11191, Myanmar \\ Corresponding Author: Khin Thet Wai \\ E-mail: khinthetwaidmr@gmail.com
}

\begin{abstract}
Ethical oversight of the Institutional Review Boards (IRB) could facilitate the beneficial impact on the conduct of implementation research in rural settings during the public health emergency of international concern (PHEIC). There is a knowledge gap in the application of implementation research ethics to uphold the impact on the planning of community surveys during the COVID-19 outbreak in remote rural settings. This study aimed to underscore the implementation research ethics principles incorporated in the IRB review practice at the Department of Medical Research, Myanmar by examining the records of three implementation research proposals submitted between April to October 2020. These records targeted the self-help groups, malaria volunteers, and nutrition-support groups who will introduce the innovative interventions in the remote rural areas, malaria-afflicted communities and the households of women with young children in their first 1,000-days respectively. Given that social, economic, and local administrative issues contribute for the acceptability, adoption, and effectiveness of interventions, the IRB has seriously considered the strategic plan for community engagement at vulnerable sites during PHEIC in addition to the contextual equipoise. It is also imperative to introduce the protective measures to mitigate the transmission among the research team members and the target audience in the COVID-19 period. Safe data collection modes without any interpersonal contact are desirable. The improved awareness of implementation research ethics principles and the applications is crucial for further capacity strengthening of IRBs as next steps to fit their reviews with PHEIC in a resource-constrained environment
\end{abstract}

Keywords: Ethical oversight, Implementation research, contextual equipoise, community engagement, public health emergency, COVID-19

\section{INTRODUCTION}

A multidisciplinary nature and the involvement of multiple stakeholders are keys to implementation research in a real-world setting. It aims for sustainable health gains resulting from 
successful interventions [1]. Throughout the implementation research process, there is a need for comprehensive and meaningful stakeholder engagement. Features of implementation research mainly include the relevance and responsiveness to local health priorities [2-3]. Along a similar vein with health policy and systems research, the Institutional Review Boards (IRB) raise several ethical issues in research design while reviewing the implementation research (IR) proposals. The Global Health Ethics Team at WHO, in collaboration with TDR, elucidated the important knowledge gap concerning the ethical implications of implementation research [4]. Ethical oversight of the Institutional Review Boards (IRB) could facilitate the beneficial impact on the conduct of implementation research in rural settings during the public health emergency of international concern (PHEIC) [5-10].

In late March 2020, Myanmar has reported its first confirmed cases followed by the effective response of the State to the public health emergency condition to curbing disease spread. Introducing non-pharmaceutical interventions coupled with the regulatory measures are in place. These interventions engender contact-tracing, testing, quarantine, mask-wearing, the prohibition of mass gathering and the official closure of religious and recreation sites, universities and colleges, schools, food establishments, the release of stay-home orders, and travel restrictions etc. Until midAugust, there were only 374 reported cases and six deaths. From then on, the number of positive cases among the tested increased up to over 1,000 every single day till the end of November 2020 according to the official reports [11]. Some of the public health interventions with or without the partnership with the International Non-Governmental Organizations (INGOs) and the development agencies temporarily halted at the beginning of the pandemic but resumed later by observing the universal precautions for mitigating COVID-19 transmission. Between April to October 2020 within the COVID-19 pandemic period, the IRB at the Department of Medical Research (DMR) in Myanmar has assessed five implementation research proposals with more than minimal risk under the full board review meetings [12]. Of these proposals, three studies included the innovative approach in rural settings through a myriad of volunteer groups in which only one study focused COVID-19 socio-behavioural aspect. Those groups reflected the most desirable form of community engagement to control non-communicable diseases, malaria and to prevent nutritional deficiencies in their specific project sites. Importantly, the selected proposals also demonstrated the unique feature of the collaborative research within the public health programs [13] launched by the Department of Public Health in partnering project management and supervision, sharing resources and technology with the International Non-Governmental Organizations (INGOs) in line with their official agreement (a memorandum of understanding) with the Ministry of Health and Sports (MoHS), Myanmar.

Particular ethical considerations in implementation research should cover about from whom and how informed consent can or should be obtained; balancing of risks and benefits that could accrue to different groups; the responsibility of researchers to address ancillary findings, especially where health systems are weak; and appropriate dissemination of results to ensure timely uptake and scale-up of successful interventions $[2,3,13]$. In times of public health emergencies such as infectious disease outbreaks, ethics guidelines for implementation research together with other forms of public health research become more stringent than before to safeguard the project team as well as the target beneficiaries [6]. Apart from the contextual equipoise, it is imperative to seek for ethical concerns linking to implementation research outcomes. As such, the focus will be on access, acceptability, reach, adoption, and utilization of health interventions and sustainability and scaled-up [14-15] within the weak rural healthcare infrastructure of the developing country amid pandemic restrictions and regulatory measures. In this connection, there is limited knowledge available to date in all parts of the world in relation to how IRBs respond to and appraise implementation research during the PHEIC with implications for further capacity building. Therefore, this study aimed to underscore the ethical challenges of implementation research proposals reviewed and considered during the COVID-19 pandemic from April to October 2020 at the IRB of the DMR. 


\section{METHODOLOGY}

We performed the case-based reviews of three IRB records of implementation research related to public health interventions using the inclusive self-help groups (ISHG), trained malaria volunteers and the nutrition support groups. For each research project reviewed at the IRB, the analysis informed the project description, and a brief account on ethical considerations without revealing the actual title of the research projects and partner organizations (INGO A, B and C) to observe anonymity and confidentiality in line with Helsinki declaration. The methodology in constructing case studies followed a previous paper [16]. Ethical challenges in this study referred to ethical issues related to implementation research that needed to take into account during the IRB review process that were difficult to deal with, in the absence of clear guidelines or known precedents [4]. The IRB (DMR) exempted the review of this research. The administrative authorities provided permission to review the records for further analysis.

\section{RESULTS}

All three research projects provided strong justification to introduce IR in the selected contexts: target beneficiaries of primary health care services in rural areas, villages targeted for malaria elimination and low-income households of women with young children in their first 1,000-days. The national counterparts of the ongoing public health programs in Myanmar planned those research in collaboration with the respective INGOs.

The proposed study designs of three implementation research projects were variable. These included a pre-test and post-test quasi-experimental intervention study without a control group (case study 1), an open step-wedged cluster-randomized controlled trial (case study 2), and a mixed-methods study with serial cross-sectional surveys (case study 3 ). Brief project descriptions were shown in text boxes 1 to 3 .

\section{Text Box 1: Brief project description of viable inclusive self-help groups in rural areas}

\section{Case study 1}

Implementation research for viable inclusive self-help groups to enhance strategies in primary health care coverage in rural households

Despite the increasing burden of non-communicable diseases (NCDs), significant gaps and inequalities in the healthcare delivery system pose as barriers in accessing and sustaining care and treatment. Furthermore, evidence of effective interventions targeting diabetes and hypertension as major NCDs in rural areas remains limited. A set of cost-effective interventions are planned at the village level in country $\mathrm{X}$ in collaboration with the INGO (A). The implementation research includes further scaling up and training of Inclusive Self-Help Group Volunteers (ISHG) to deliver health knowledge through health promotion sessions. Also, they will do basic screening service to refer to the primary health care (PHC) facilities, as well as to deliver ancillary services for the vulnerable groups and to establish the linkage with their closest PHC facilities.

\section{Ethical considerations}

In case-study 1, community engagement through intensive advocacy meetings with village authorities, influential persons, and the rural health centre staff are critical for trust-building to ISHG volunteers, and for creating mutual respect between them and PHC workers at the village level. The guidelines of the MoHS to prevent COVID-19 transmission should be in place when conducting the advocacy meetings for community engagement. Moreover, community consent is the appropriate choice rather than individual informed consent which should initiate from the inception of the project. Importantly, timely referral to the nearest PHC facilities for ancillary care 
is crucial in line with instructions of MoHS after detecting any abnormality in screening that might include suspected COVID-19 cases. Expected benefits such as an increase in health knowledge, and understanding of feasibility, acceptability, and effectiveness of the proposed intervention will outweigh the risks thus encouraging the access of vulnerable households in the rural community to NCD-related primary health care services. During the process and end-evaluation, arrangements for data curation, data sharing, and data confidentiality between implementing partners requires careful deliberation particularly in the COVID-19 outbreak period.

\section{Text Box 2: Brief project description of malaria volunteer groups in an endemic rural setting}

\section{Case study (2)}

Implementation research to strengthen the malaria elimination strategies in the rural communities of country X

Despite the rapid expansion of the malaria program coverage, an emergence of artemisinin resistance and the existence of pocket areas of residual infection deter the National Malaria Control Programmes (NMCP) to meet the targets for malaria elimination goal by 2030. In this context, the NMCP in country X partnered with the INGO (B) to identify and implement the new package of malaria interventions. The innovative community-based integrated model will encompass strategic interventions through the deployment of trained malaria volunteers. Project villages are to be included by a step-wedged pattern that differs from the traditional model. The new model focuses on the expansion of the scope of activities of malaria volunteers as opposed to the conventional model. It will include the additional screening for dengue, filariasis, TB and HIV/AIDS. Moreover, appropriate referral service will provide more opportunities for them to test malaria by RDT among rural householders who are not in easy access due to their mobile nature of livelihoods.

\section{Ethical considerations}

For case-study 2, it is the responsibility of the health systems while engaging and working with vulnerable populations in villages with the potential for pockets of residual malaria and prone not only to the risks of outbreaks but also to other vector-borne diseases such as dengue and filariasis and major killers like TB and HIV/AIDS. There is the uncertainty of how the proposed intervention package will be accepted and be effective within the given context that is 'contextual equipoise'. While implementing the intervention, the responsibility for the provision of ancillary care for illnesses other than malaria is required. Expected benefits such as an increase in understanding of feasibility, acceptability and improved access to the proposed intervention will outweigh the risks to enhance the malaria elimination activities. For trust-building to newly equipped malaria volunteers, community engagement through intensive advocacy meetings with village authorities, influential persons and the rural health centre staff is critical. To avoid the invasion of privacy and discomfort by interviewing the community members and village authorities by the trained malaria volunteers of the same locality, separately trained interviewers by the research team should perform the recruitment and taking the informed consent and the evaluation of the proposed intervention activities. In times of public health emergency, it would be essential for malaria volunteers to practice the universal precautions to prevent COVID-19 transmission. Data sharing and data confidentiality process between implementing partners of the proposed intervention requires careful deliberation. 
Text Box 3: Brief project description of nutrition support groups in poverty stricken rural sites

Case study (3)

The add-on mixed methods study of implementation research by the nutrition support groups concerning COVID-19 risk perceptions and risk mitigation behaviours in households of women with young children in their first 1000-days

The poverty-stricken rural communities of Regions A and B of Country X have reported a high prevalence of malnutrition in under-five children due to low exclusive breastfeeding, improper infant and young child feeding practices, lack of dietary diversity, and food insecurity as an impact of insufficient agricultural resources. As guided by the National Nutrition Center, the INGO (C) has introduced the "Enhanced Homestead Food Production Program" with emphasis on the behavioural change communication aimed at correct feeding practices for young children in their first 1000-days combined with the arrangements of adequate water supply for hygiene and sanitation in these areas. During the COVID-19 pandemic period, the nutrition support groups of INGO (C) have distributed preventive materials to project households. They would like to assess the risk perceptions of householders and their risk mitigation practices for COVID-19 as an addon study. The study covered the EHFP program beneficiaries and the respective health and local authorities and purposively selected community members. Due to the semi-lockdown conditions, the research team planned two-rounds of quantitative and qualitative data collections through mobile phones.

\section{Ethical considerations}

In case-study 3, community engagement through comprehensive advocacy meetings to be arranged for village authorities and other influential persons is critical in the enhancement of trustbuilding between the nutrition support groups and the community regarding the use of COVID19 preventive measures and to avoid confusion between the on-going nutrition project and the addon research study using mixed methods. Since the researchers have planned to use the phone-based data collection, there is an unequal chance of selection for the households without mobile phone access. Also, passing on the phone among the household members might pose increased health risks that might outweigh the benefits. The data collection planned by the responsible staff of the implementing organization of the particular locality might create undue influence and conflict of interests. The validity of verbal consent by the selected respondents during the stressful time is questionable. The research team should inform the respondents about their right in refusal to participate in this research that could not affect the program benefits obtained. Respondents could receive mobile money for their phone bills, not more than three USD is considered appropriate as an incentive for participation which is equivalent to the daily minimum wages as of 2019 in Myanmar. Keeping the survey short by avoiding lengthy and ambiguous questionnaires is to reduce the cost, time, and frequency of phone calls as well as to avoid the discomfort of research participants. Data confidentiality and data sharing among the implementing partners is highly desirable to support the dissemination and utilization of salient findings to the target beneficiaries that aims to support sustainability.

\section{DISCUSSION}

The claim of this present study mainly addresses the role of IRBs to perform thorough scrutiny in implementation research ethics during the pandemic period in remote rural settings without adequate healthcare infrastructure to achieve quality ethics oversight. One study from India provided a strong evidence to foster ethical biomedical and health research during the COVID-19 pandemic [17]. Each proposal fulfilled the specific components in designing the implementation research study [4]. Table 1 summarized the specific ethical challenges encountered in each proposal. 
Table 1: Summary of ethical challenges in implementation research during COVID-19 pandemic in Myanmar (April to October 2020)

\begin{tabular}{|l|c|c|c|}
\hline Ethical challenges & $\begin{array}{c}\text { Case 1 } \\
\text { Inclusive } \\
\text { self-help groups for } \\
\text { primary health care }\end{array}$ & $\begin{array}{c}\text { Case 2 } \\
\text { Malaria } \\
\text { volunteers }\end{array}$ & $\begin{array}{c}\text { Case 3 } \\
\text { Nutrition support } \\
\text { groups } \\
\text { (add-on mixed } \\
\text { methods COVID-19 } \\
\text { perception survey) }\end{array}$ \\
\hline Study design & - & $*$ & - \\
\hline Study context/relevance & $*$ & $*$ & $*$ \\
\hline Acceptability of intervention & $*$ & $*$ & - \\
\hline Equipoise & - & $*$ & $*$ \\
\hline Sustainability & $*$ & $*$ & $*$ \\
\hline Incentives & $*$ & $*$ & - \\
\hline Quality control & $*$ & $*$ & $*$ \\
\hline Group/community consent & $*$ & $*$ & $*$ \\
\hline $\begin{array}{l}\text { Selection of community } \\
\text { representatives }\end{array}$ & $*$ & $*$ & $*$ \\
\hline Ancillary care & $*$ & $*$ & $*$ \\
\hline
\end{tabular}

Source: Adapted from- Ethics in implementation research. Facilitator's guide. Geneva: World Health Organization; 2019. *- denotes the specific ethical consideration

Ethical challenges in implementation research particularly in time of PHEIC as noted in three case records varied in terms of the study design, contextual equipoise, recruitment, and community consent. There is an argument on the necessity of special consideration on ethical issues in the design and conduct of stepped-wedge cluster-randomized trials in both high- and low-resource settings in terms of the justification for using the design, and the classification of the study as research or non-research [18-19]. Unlike the intervention study without a control group (case study 1) and a mixed-methods study with rounds of cross-sectional surveys (case study 3 ), the stepwedged cluster randomized controlled trial (SW-CRT) (case study 2) raised some ethical considerations together with methodological constraints. In a low-resourced setting, the application of SW-CRT was feasible and relevant particularly related to a delay in rolled out of the intervention package for malaria elimination to some control villages. Nevertheless, in terms of risk-benefit assessment, the risks in delay should be considered as important as the intervention is perceived to be superior compared to the standard of care [18].

Adequate protection of human research participants in selected villages to mitigate ethical challenges relied upon the application of specific ethical guidelines by the research team as suggested by the IRB. Furthermore, the IRBs should scrutinize for the underlying reason in the choice of clusters (villages) as the first step to introduce interventions by targeting the mostly needed ones as described in case study 2 . However, SW-CRTs are less efficient, more prone to bias, and expose more people to harm than conventional RCTs. Practical and logistical reasons for using a CRT or SW-CRT should be weighed against the possible advantages of more rigorous methods such as conventional RCTs [18]. In addition, observing the contextual equipoise of the proposed implementation research is the real challenge for IRB members due to uncertainties and unexpected events like disasters and public health emergencies $[5,6,9]$. It is particularly true in case study 2 .

Given that social and economic and local administrative issues contribute to the acceptability and effectiveness of interventions in all case studies in this research, the IRB has seriously considered the strategic plan proposed by the research teams for community engagement in their project sites during public health emergency where vulnerable communities existed with health inequities [6, 10]. The IRBs need thorough scrutiny of the transparent recruitment procedures fit to pandemic restrictions as well as the informed consent forms to observe autonomy [20] either in the form of 
community consent to reach vulnerable households or the individual ICF used during the initial, mid-term, and end-evaluation surveys as noted in case study 3 with an addendum of two rounds of COVID-19 perceptions survey. Balancing risks and benefits and plans to provide ancillary services by the implementing partners or the appropriate referral service could pave the way to the ethical conduct of implementation research [2,21]. Data sharing platforms varied by the source of data and methods of data collection thus creating difficulty to maintain data confidentiality also noted by other studies [11].

In the context of the COVID-19 pandemic, IRBs need to consider specific elements of research proposals during the ethics review process. An ethics oversight should focus on the compliance of each and every study with the international and local laws, rules, and guidelines for responses towards disasters and public health emergencies and strict adherence during the actual conduct $[8$, 22]. Moreover, during the unprecedented COVID-19 pandemic period, it was imperative to introduce protective measures/strategies to safeguard against and to mitigate the transmission and risk of acquisition of infection for both research team members and the target audience [6]. Optimizing uptake of evidence-based interventions such as using personal protective equipment is mandatory already included in the ethical considerations of the provided case studies in this research. The adherence to COVID-19 restrictions enhances the use of safe data collection modes without any interpersonal contact such as using mobile applications, and telephone interviews to avoid undue risks as noted across all case studies. Also, ethics reviews should focus on transparency during recruitment $[9,23]$. The arrangements for ethical dissemination of salient findings by virtual means during the PHEIC mainly depend on the complex nature of implementation research being based upon multi-stakeholders' involvement at different levels of the healthcare system [7].

\section{Strengths and limitations}

This is the first study reported from the IRB of a developing country where there is successful planning for implementation research projects of various designs in rural communities during the pandemic period. All of these proposals require appropriate modifications for ethical conduct during the period of PHEIC after paving their way through the IRB review process. However, in this study, we could not explore ethical issues/challenges involved in all types of implementation research designs.

\section{Conclusions}

Reviewing and appraisal of the implementation research proposals by IRBs in low-constrained settings is not an easy task. Current IRB review forms may require revisions to fit with epidemic ethics as well as for an implementation research incorporating public health interventions. Besides, IRBs should attempt for an after-review process and remote monitoring during the pandemic period by guiding the researchers to submit the progress reports regularly for the fulfilment of quality ethical oversight. The formidable challenges raised in implementation research ethics are manifold that requires attention to develop specific ethics framework and indicators in low resource settings. The evidence informed IRB policy and procedures for implementation research ethics is a necessity to improve awareness and meaningful applications. It is crucial for further capacity strengthening of IRBs as next steps to fit their reviews with PHEIC in a resourceconstrained environment.

\section{REFERENCES}

1. Hales S, Lesher-Trevino A, Ford N, Maher D, Ramsay A, Tran N. Reporting guidelines for implementation and operational research. Bull World Health Organisation 2016;94(1):58-64

2. Macklin R. Ethical challenges in implementation research. Public Health Ethics 2014;7(1):86-93.

3. David H. Peters, Nhan T. Tran, Taghreed Adam. Implementation research in health: a practical guide. Alliance for Health Policy and Systems Research. World Health Organization.2013.

4. Ethics in implementation research. Facilitator's guide. Geneva: World Health Organization; 2019. Licence: CC BY-NC-SA 3.0 IGO. 
5. World Health Organization. Guidance for managing ethical issues in infectious disease outbreaks. Geneva. Switzerland 2016.

6. WHO. Ethical standards for research during public health emergencies: Distilling existing guidance $\begin{array}{llllll}\text { to } & \text { support } & \text { COVID-19 } & \mathrm{R} & \& & \mathrm{D}\end{array}$ https://apps.who.int/iris/bitstream/handle/10665/331507/WHO-RFH-20.1-eng.pdf.

7. WHO. Guidance for research ethics committees for rapid review of research during public health emergencies. 2020. file:///C:/Users/User/Downloads/9789240006218-eng\%20(3).pdf

8. Research in Global Health Emergencies: Ethical Issues. Nuffield Council on Bioethics, 2020.

9. Yeoh KW, Shah K. Research ethics during a pandemic (COVID-19). International Health 2020:12.

10. McGuire AL, Aulisio MP, Davis FD, Erwin C, Harter TD, Jagsi R, Klitzman R, Macauley R, Racine E, Wolf SM, Wynia M, Wolpe PR \& The COVID-19 Task Force of the Association of Bioethics Program Directors (ABPD) Ethical Challenges Arising in the COVID-19 Pandemic: An Overview from the Association of Bioethics Program Directors (ABPD) Task Force. Am J Bioethics 2020;20(7):15-27.

11. MoHS. COVID-19 Surveillance Dashboard. Ministry of Health and Sports, Myanmar 2020. https://www.mohs.gov.mm

12. Database of the Institutional Review Board, Department of Medical Research. 2020.

13. Wai KT, Aye N.S. Collaborative Research in Health Sector. In: Wai KT, Aung W, Oo ZZ. (eds). Principles of Responsible Conduct of Research and Research Ethics: A Handbook. Department of Medical Research, Ministry of Health and Sports, Myanmar, 2020. https://www.mohs.gov.mm

14. Win HH, Wai KT. Implementation Research Ethics. In: Wai KT, Aung W, Oo ZZ. (eds). Principles of Responsible Conduct of Research and Research Ethics: A Handbook. Department of Medical Research, Ministry of Health and Sports, Myanmar, 2020. https://www.mohs.gov.mm

15. Proctor E., Silmere H, Raghavan R, Hovmand P, Aarons G, Bunger A, et.al. Outcomes for implementation research: conceptual distinctions, measurement challenges, research agenda. Adm Policy Mental Health 2011;38:65-76.

16. Gopichandran, V., Luyckx, V.A., Biller-Andorno, N. et al. Developing the ethics of implementation research in health. Implementation Sci 2016;11:161.

17. Kumar NK, Muthuswamy V. Fostering ethical biomedical and health research in India during the COVID-19 pandemic. Research Ethics 2020;16(3-4):1-10.

18. Joag, K., Ambrosio, G., Kestler, E. et al. Ethical issues in the design and conduct of stepped-wedge cluster randomized trials in low-resource settings. Trials 2019;20(703).

19. Hemming K, Taljaard M. Reflections on modern methods: when is a stepped-wedge cluster randomized trial a good study design choice? Int J Epidemiol 2020;10;1043-52.

20. van der Graaf, R., Cheah, P.Y. Ethics of alternative trial designs and methods in low-resource settings. Trials 2019;20:705.

21. Leadbeater, B.J., Dishion, T., Sandler, I. et al. Ethical Challenges in Promoting the Implementation of Preventive Interventions: Report of the SPR Task Force. Prev Sci 2018;19:853-65.

22. Tindana PO, De Vries $J$ and Kamuya $D$. Ethical challenges in community engagement practices in research during the COVID-19 pandemic in Africa. AAS Open Res 2020;3:23.

23. Mathur, R. Ethics preparedness for infectious disease outbreaks research in India: a case for novel coronavirus disease 2019. Indian J Med Res 2020;151(2 \& 3):24-31.

Acknowledgements: Authors would like to extend their heartfelt thanks to Professor Henry Silverman, University of Maryland Baltimore (UMB) for his initial review, suggestions and kind edits of the initial drafts of this paper.

This paper has been accepted for the poster presentation at the Virtual Workshop on "Advancing Research Ethics in Asia and the Pacific" (17-18 November 2020) organized by the University of Maryland Baltimore, USA.

Conflict of interest: Nil 